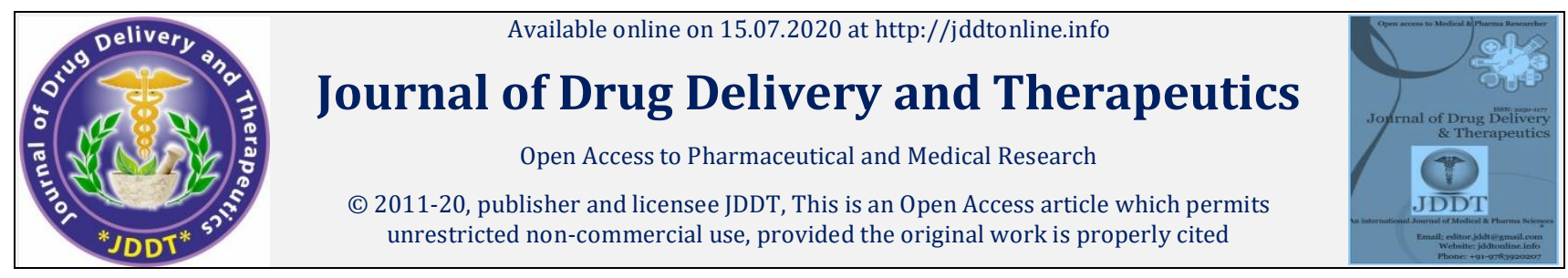

Open@Access

Research Article

\title{
A Design of Experiment Approach for Optimization and Characterization of Clarithromycin Ternary System Using Spray Drying
}

\author{
Nikhil Arun Shete1*, Vishwajeet M. Swami2 ${ }^{2}$ Avinash Chaudhari³ ${ }^{3}$ Prachi Khabiya ${ }^{4}$, \\ 1 Master of Pharmacy (Pharmaceutics), Dr D. Y. Patil College of Pharmacy, Akurdi Pune, India \\ 2 Master of Pharmacy (Pharmaceutics), RJSPM'S Institute of Pharmacy Pune, India \\ ${ }^{3}$ Master of Pharmacy (Pharmaceutics), Indira College of Pharmacy Tathawade, Pune, India \\ 4 Master of Pharmacy (Chemistry), AISSMS College of Pharmacy, Sangamvadi, Pune, Maharashtra 411001, India
}

\begin{abstract}
The aim of present work was to characterize Clarithromycin (CLT), Polyvinyl pyrrolidone K30 (PVP K30) and Hydroxypropyl $\beta$-cyclodextrin (HPB) ternary system so as to check the effect of complexation on solubility of CLT. Physical mixtures of a drug and polymers in several weight ratios $(1: 1,1: 2)$ were prepared to check the effect of individual polymers on solubility of CLT. Spray drying method was accustomed investigate the combined effect of PVP K30 and HPB on Drug release (DR), Dissolution efficiency (DE) and mean dissolution time (MDT) of CLT. For the preparation and optimization of ternary system the Design of experiment (DoE) was used. Drug polymer interactions were analyzed with Fourier transform infrared spectroscopy (FTIR), Differential scanning calorimetry (DSC), X-ray diffraction (XRD) and particle size analysis. Results of solubility study suggested that there was significant increase in solubility of CLT with increase within the concentration of PVP K30 and HPB $\left({ }^{*} \mathrm{p}<0.05\right)$. This may be thanks to the solubilizing effect of PVP K30 and sophisticated formation of CLT with HPB. Various combinations of PVP K30 and HPB prepared using DoE approach by spray drying method showed greater solubility of CLT than its physical mixtures $\left({ }^{*} p<0.05\right)$. Results of FTIR, DSC, XRD and particle size analysis revealed the interaction between CLT, PVP K30 and HPB. This suggested formation of amorphous ternary system with mean particle diameter within the range of $312 \pm 1.35 \mathrm{~nm}$. Combine use of PVP K30 and HPB with DoE approach was an efficient tool for formulating ternary system of CLT.
\end{abstract}

Keywords: Clarithromycin, Spray drying, polyvinyl pyrrolidone K30, Hydroxypropyl $\beta$-cyclodextrin, Design of experiments, Ternary system.

Article Info: Received 21 April 2020; Review Completed 12 June 2020; Accepted 27 June 2020; Available online 15 July 2020

Cite this article as:

Shete NA, Swami VM, Chaudhari A, Khabiya P, A Design of Experiment Approach for Optimization and Characterization of Clarithromycin Ternary System Using Spray Drying, Journal of Drug Delivery and Therapeutics. 2020; 10(3-s):211-220 http://dx.doi.org/10.22270/jddt.v10i3-s.4185

*Address for Correspondence:

Nikhil Arun Shete, Master of Pharmacy (Pharmaceutics), Dr D. Y. Patil College of Pharmacy, Akurdi Pune, India

Abbreviations: CLT- Clarithromycin, DSC- Differential scanning calorimetry, XRD- X-ray diffraction, FTIR- Fourier transform infrared spectroscopy, DR- Drug release, DE- Dissolution efficiency, MDT- mean dissolution time, DoE- Design of experiment, CDs- cyclodextrins, PVP- polyvinyl pyrrolidone.

\section{INTRODUCTION}

For improving drug dissolution rate, formulation of drugs with cyclodextrins (CDs) has demonstrated to be a powerful tool in the pharmaceutical field $1-3$. For improving drug dissolution by complexation, CDs have been extensively used in pharmaceuticals. CDs modifies physicochemical properties of the guest molecule such as solubility and stability by molecular encapsulation ${ }^{4-6}$. Hydrophilic exteriors of CDs imparts (or their complexes) water solubility and hydrophobic interior of it able to accommodate a guest molecule within the cavity ${ }^{7}$. Factors responsible for behavior of CDs in these complexes are proximity of charge to the CDs cavity, charge state of the CDs, steric factors, nature of the drug, co-solvent effects and temperature ${ }^{8}$. This strategy reduces the cost of a drug and enhances its bioavailability. This further reduces dose and side effects of drug 9 -10.

Clarithromycin (CLT), is an macrolide antibiotic used to treat respiratory tract infections, skin infections and Helicobacter pylori infections. CLT acts by the inhibition of bacterial protein biosynthesis. CLT is having low solubility and high permeability so it belongs to BCS class II drugs. Therefore, the enhancement of dissolution rate and solubility is 
expected to significantly improve its bioavailability and reduce its side effects.

Spray drying is an ordinarily utilized technique for drying a fluid feedthrough hot gas. Regularly, this hot gas is air however sensitive materials, for example, pharmaceuticals and solvents like ethanol require oxygen-free drying and nitrogen gas is utilized. The fluid feed changes relying upon the material being dried and is not constrained to food or pharmaceutical products and might be a solution, colloid or a suspension. This procedure of drying is a one-step quick procedure and eliminating extra processing. Spray drying includes the atomization of a liquid feedstock into a spray of droplets with hot air in a drying chamber. The sprays are delivered by either rotary (wheel) or nozzle atomizers. Evaporation of moisture from the droplets and formation of dry particles continue under controlled temperature and airflow conditions. The powder is released persistently from the drying chamber. Working conditions and drying configuration are chosen by the drying qualities of the product and the powder particular ${ }^{11}$.

Systematic design of experiment (DoE) approach is extensively practiced in the development of drug delivery devices. Advantage of this approach is that it requires few experimental trials to achieve an optimum formulation. Optimization using DoE is a cost-effective analytical tool, quick and best solution to a particular defined problem. This approach provides an ability to explore and determines ranges of polymer ${ }^{12,13}$.

Therefore, the aim of present study was to develop spray dried ternary system of CLT-polyvinyl pyrrolidone K30 (PVP K30), hydroxypropyl $\beta$ cyclodextrin (HPB) by DoE approach, which ultimately improves dissolution profile and solubility of drug. Central composite design (CCD) was used for the preparation of spray dried ternary system with the help of a software, Design expert.

\section{MATERIALS AND METHODS}

\section{MATERIALS:}

Carithromycin (CLT) and hydroxypropyl $\beta$ cyclodextrin (HPB) were obtained from Alembic pharmaceuticals Ltd., Vadodara, polyvinylpyrrolidone K30 (PVP K30) were obtained from Lupin research park, Pune, India. All other reagents and chemicals were of analytical grade.

Equipment Used:

\begin{tabular}{|l|l|l|}
\hline Sr no. & Equipment/ instrument & Make and Model No. \\
\hline 1 & Electronic Balance & METLER TOLEDO, ME 204 \\
\hline 2 & Spray Dryer & Labultima LU-222 \\
\hline 3 & Mechanical Stirrer & REMI RQ 5 Plus \\
\hline 4 & USP Dissolution Apparatus Type II & SCIENTIFIC BZ 200 \\
\hline 5 & FTIR Spectrophotometer & SHIMADZU AFFINITY 1-8400 S \\
\hline 6 & X-Ray diffractometer & PW 3710 BASED \\
\hline 7 & X-ray powder diffraction (XRD) & SII Nanotechnology (SIEKO)-6220 \\
\hline 8 & Particle size Analyser & $\begin{array}{l}\text { Malvern Zetasizer ZS90 (Malvern Instruments, } \\
\text { Worcestershire, UK) }\end{array}$ \\
\hline
\end{tabular}

\section{METHODS}

\section{Design of Experiments (DoE)}

Design Expert V10 software was used for designing of experiments. The amounts of HPB (A) and PVP K-30 (B) were selected as experimental factors and studied at three levels each. A CCD with $\alpha=1$ was employed as per standard protocol12,13. The central point $(0,0)$ was studied in quintuplicate. All other formulation ingredients and processing variable were kept constant throughout the study. As depicted in Table 1 Thirteen experimental runs with different combinations of factors were obtained by design expert software. Percent drug release $(\% \mathrm{R})$, dissolution efficiency (\%DE) and mean dissolution time (MDT) were taken as response variables.
Table 1: Central composite design.

\begin{tabular}{|c|c|c|}
\hline Run & HPB (A) mg & PVP K30 (B) mg \\
\hline 1 & 250 & 125 \\
\hline 2 & 300 & 50 \\
\hline 3 & 250 & 125 \\
\hline 4 & 250 & 125 \\
\hline 5 & 250 & 231.066 \\
\hline 6 & 200 & 200 \\
\hline 7 & 250 & 125 \\
\hline 8 & 179.289 & 125 \\
\hline 9 & 320.711 & 125 \\
\hline 10 & 300 & 200 \\
\hline 11 & 250 & 18.934 \\
\hline 12 & 250 & 125 \\
\hline 13 & 200 & 50 \\
\hline \multicolumn{2}{|c|}{ HPB: Hydroxypropyl $\beta$-cyclodextrin; PVP: Polyvinyl } \\
\hline \multicolumn{2}{|c|}{ pyrrolidone } \\
\hline
\end{tabular}




\section{Preparation of solid dispersion or inclusion complexes}

Different methods namely Kneading and Spray drying method were used for preparation of drug and polymers complexes in various concentrations as shown in Table 1.

\section{Kneading method}

HP- $\beta$-CD and PVP K30 were added to the mortar, and small quantities of $50 \% \mathrm{v} / \mathrm{v}$ ethanol were added while triturating to get slurry-like consistency. Then slowly the drug was added into the slurry, and trituration was continued further for $1 \mathrm{hrs}$. The slurry was then air-dried at $25^{\circ} \mathrm{C}$ for $24 \mathrm{hrs}$. Pulverized, and passed through the sieve using \# 100 and stored in a dessicator over fused calcium chloride ${ }^{13}$.

\section{Spray drying method}

The accurate weight of CLT was dissolved in ethanol. Required amounts of PVP K30 and HP- $\beta$-CD were dissolved in purified water. The solution was further mixed with constant stirring and then spray dried (Labultima LU-222). The drying conditions were as follows: inlet temperature $120^{\circ} \mathrm{C}$; outlet temperature $70^{\circ} \mathrm{C}$; aspirator $80 \%$; feed rate $3 \mathrm{mg} / \mathrm{ml}^{14}$.

\section{In-vitro Drug release study:}

Drug release studies were performed by employing the USP dissolution apparatus (Type II.) Solid dispersions powder equivalent to $250 \mathrm{mg}$ of Clarithromycin \& Plain Drug were inserted in a dissolution vessel containing $900 \mathrm{ml}$ of dissolution medium maintained at $37 \pm 0.5^{\circ} \mathrm{C}$ and stirred at $50 \mathrm{rpm} .5 \mathrm{ml}$ of Samples were collected periodically and replaced the same amount by fresh dissolution medium. Withdrawn samples were filtered through Whatman filter paper. The concentration of both pure clarithromycin \& Solid Dispersion was determined spectrophotometrically at $210 \mathrm{~nm}$.

\section{Parameters for dissolution study}

Dissolution efficiency (DE) is defined as the area under dissolution curve (y) up to certain time $t$, express as a percentage of the area of rectangle describe by $100 \%$ dissolution in the same time ${ }^{15}$.

Mean dissolution time (MDT) is another parameter that describes the rate of dissolution of a drug. MDT value is used to compare release rate of different formulations and it represents the drug release retarding efficiency of polymers used 16 .

\section{Statistical analysis}

\% Drug release, \% DE and MDT were calculated using PCP Disso V3 software. Data of \% Drug release, \% DE and MDT were fed to design of experiment software (DoE) as response variables. To generate various models, multivariate linear regression was used. Central composite design (CCD) was used for preparing different combinations of drug and carriers. For testing the significance and validity of the models, analysis of variance (ANOVA) was applied.

\section{Flow property measurement}

Pure drug and prepared solid dispersions were evaluated for flow properties. Bulk density, tap density, angle of repose, compressibility index and Hausner's ratio were calculated to study flow properties of plain CLT and optimised ternary system of CLT, HPB and PVP K30.

\section{Characterization of plain drug and prepared solid dispersion}

Plain drug and prepared solid dispersion were characterized by differential scanning calorimetry (DSC), X-ray diffraction (XRD) and Fourier transform infrared spectroscopy (FT-IR).

\section{FTIR Spectroscopy}

IR Spectroscopy of Optimized formulation was performed by using FTIR Spectrophotometer (SHIMADZU AFFINITY 1$8400 \mathrm{~S}$ ). The spectra were scanned over the wavelength region of 4000 to $400 \mathrm{~cm}-1$ at resolution of $4 \mathrm{~cm}-1$. The procedure contained dispersing the samples in $\mathrm{KBr}$ and compressing into discs by applying a pressure of 5 tons for 5 min in a hydraulic press. The pellet was placed in the light path to obtain the spectrum.

\section{XRD Study of optimized formulation}

X-ray diffraction patterns of Optimized Formulation were traced by employing an X-Ray diffractometer (PW 3710 BASED) using Ni filtered Cuk $(\alpha)$ radiations, a voltage of 35 $\mathrm{kV}$, current of $30 \mathrm{~mA}$ and receiving slit of 0.2 In. Samples were analyzed over a $2 \theta$ range of 5-700 and 5-500 for stability interpretation. The range and the chart speed were 2 X 103 CPS and $10 \mathrm{~mm} /$ degree $2 \theta$, respectively.

\section{DSC Study of optimized formulation}

Thermo grams of the optimized formulation was studied on a TA instrument model SII Nanotechnology (SIEKO)-6220. An unfilled aluminum pan was used as a reference. DSC measurements were performed at a heating rate of $10^{\circ} \mathrm{C} / \mathrm{min}$ from 25 to $350^{\circ} \mathrm{C}$ using aluminum sealed the pan. The sample size was maintained about $5 \mathrm{mg}$ for each measurement. During the analysis, the sample cell was purged with nitrogen gas.

\section{Particle size analysis:}

The particle size analysis of the selected formulation was performed using Malvern Zetasizer ZS90 (Malvern Instruments, Worcestershire, UK) and laser diffraction beam length $2.40 \mathrm{~mm}$, range lens of $300 \mathrm{RF} \mathrm{mm}$ and at $14.4 \%$ obscuration. The mean diameter of the formulation were recorded.

\section{RESULT AND DISCUSSION}

\section{In- vitro drug release study}

The release profiles of Clarithromycin by Kneading method and Spray drying method were shown in (figure 1, and 2). From the comparative study between two methods i.e. Kneading method and Spray drying method; it can be noted that Spray drying method is superior in increasing dissolution of Clarithromycin (Figure 3). It showed that the inclusion complex of Clarithromycin (Run 7) prepared by the High speed homogenization method released $90.80 \pm 2.67 \%$ drug in 60 min and also inclusion complex of Clarithromycin (Run 7) prepared by the Kneading method released $70.74 \pm 2.12 \%$ drug in 60 min. Pure drug exhibited the release of only $25.66 \pm 0.77$ in $60 \mathrm{~min}$. It was evident that the complex exhibited the faster dissolution rate than Clarithromycin alone. The increase in Clarithromycin dissolution rate through their inclusion complexes might be due to several reasons like the formation of soluble complex, the amorphization of drug and the reduction of particle size, which consequently leads to the better wettability. 


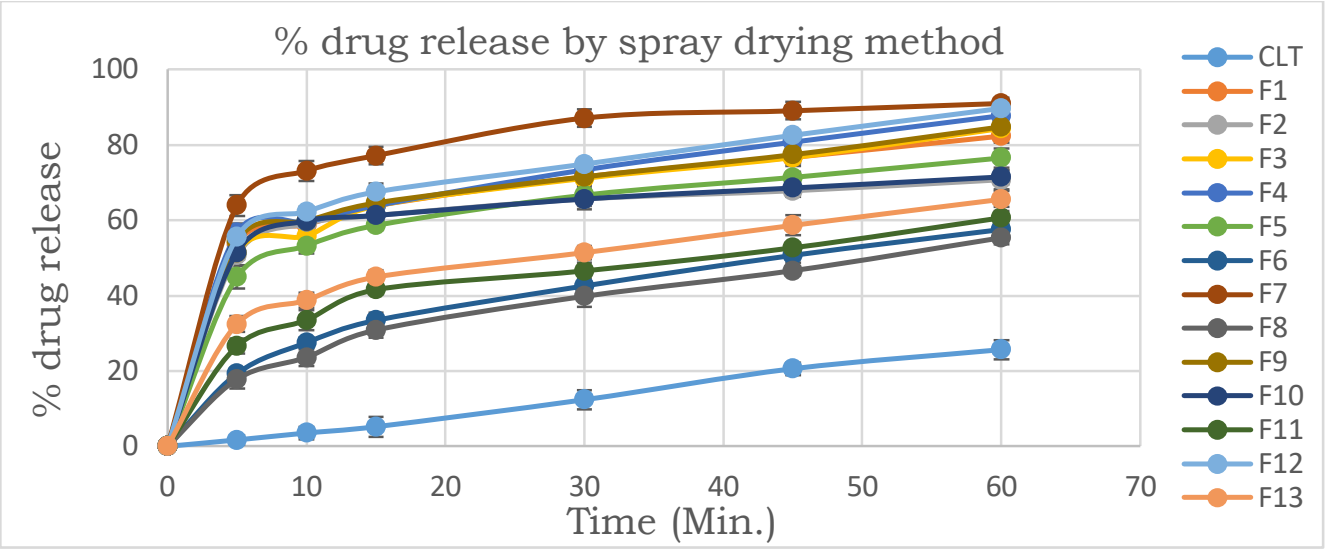

Figure1 Percent drug release of CLT by Spray drying method, Data given as mean \pm S.D. (n=3)

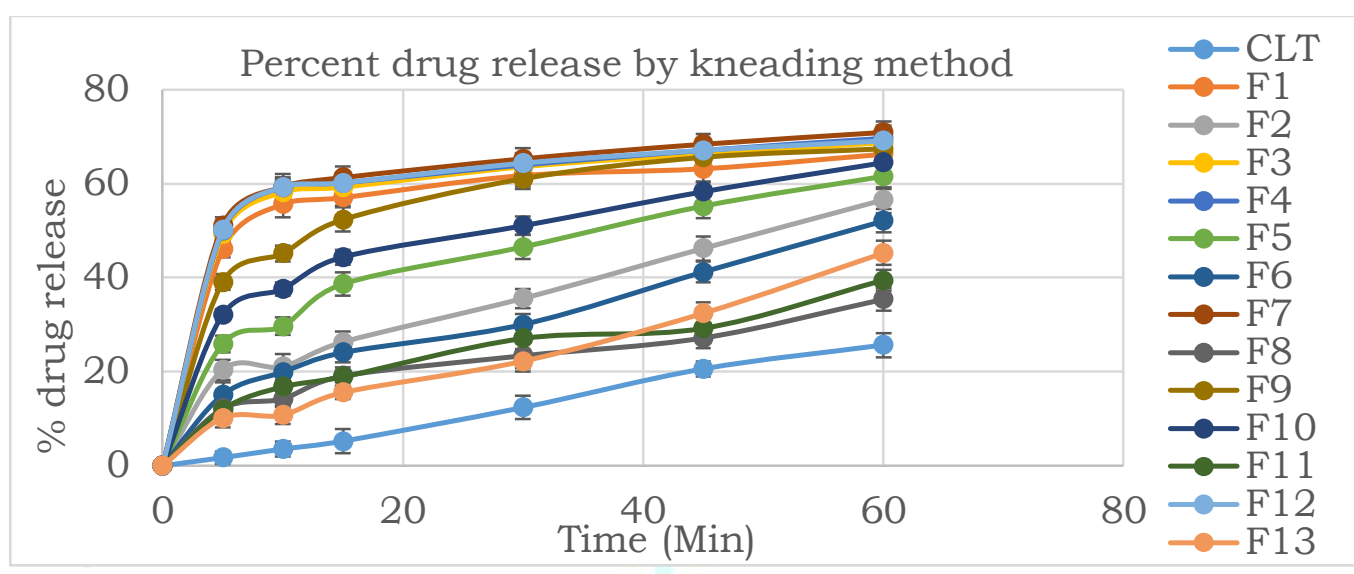

Figure 2: Percent drug release of CLT by Kneading method, Data given as mean \pm S.D. (n=3)

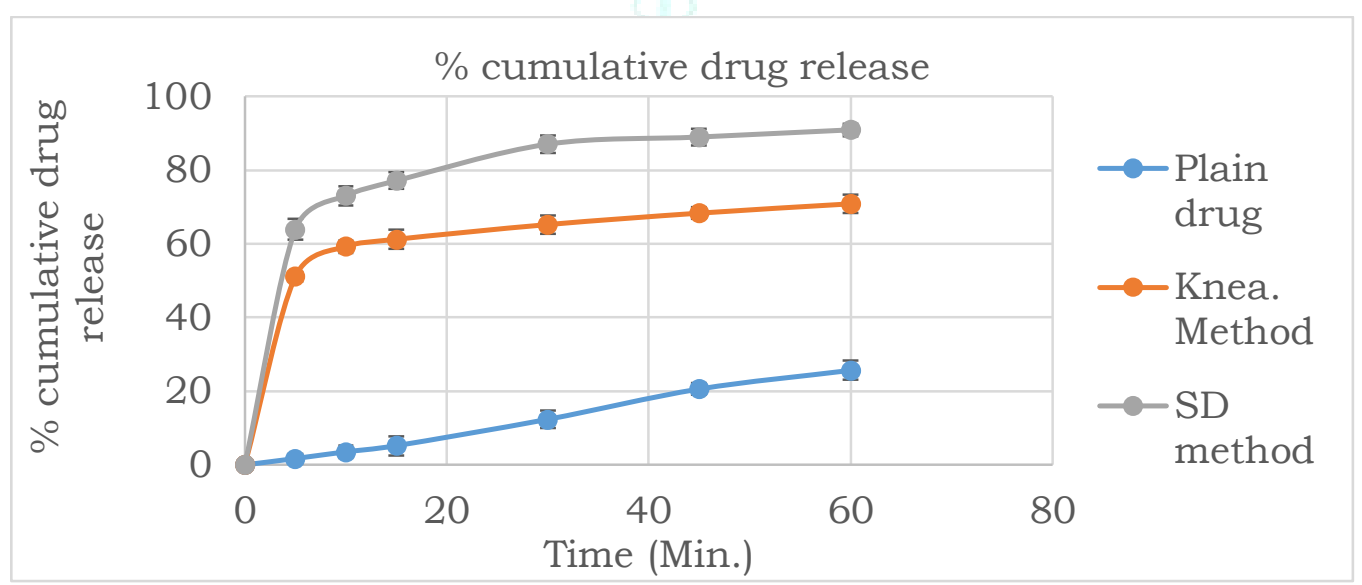

Figure 3: Comparative study of Percent drug release of CLT from optimized formulation (Run 7) Of Kneading method and Spray drying method respectively., Data given as mean \pm S.D. $(n=3)$

\section{Statistical analysis}

Table 2 depicts overall dissolution efficiency at $60 \mathrm{~min}$. and mean dissolution time of Kneading method and Spray drying method samples at $\mathrm{pH}$ 5.5. These results revealed that Spray dried clarithromycin products in presence of various combinations of HPB and PVP K-30 shows greater solubility compared to Kneading method and Spray dried method samples. The results were analysed by ANOVA $\left({ }^{*} \mathrm{p}<0.05\right)$. In case of Spray dried method, (Run 7) showed maximum dissolution efficiency of $79.68 \%$ and mean dissolution time of $7.65 \mathrm{~min}$. Dissolution efficiency and mean dissolution time of Kneading method sample (Run 7) were found to be $61.56 \%$ and $7.31 \mathrm{~min}$. respectively; and which is possibly due to highest concentration of HPB that forms complex with Clarithromycin, reduction in drug particle size, formation of hydrophilic layer over drug particles by PVP K30 and formation of inclusion complex by HPB with drug. DSC and XRD results also confirmed the formation of amorphous ternary system. 
Table 2: Dissolution efficiency (DE) and Mean dissolution time (MDT) of Kneading method and Spray drying method.

\begin{tabular}{|l|l|l|l|l|}
\hline Run & \multicolumn{2}{|l|}{ Kneading method } & \multicolumn{2}{l|}{ Spray drying method } \\
\hline & \% DE & MDT & \% DE & MDT \\
\hline 1 & 57.46 & 7.95 & 67.41 & 11.23 \\
\hline 2 & 35.35 & 22.48 & 61.56 & 7.78 \\
\hline 3 & 59.88 & 7.69 & 67.05 & 12.31 \\
\hline 4 & 60.59 & 7.78 & 69.80 & 12.27 \\
\hline 5 & 44.35 & 17.64 & 61.01 & 12.16 \\
\hline 6 & 31.24 & 23.98 & 38.89 & 19.45 \\
\hline 7 & 61.56 & 7.31 & 79.68 & 7.65 \\
\hline 8 & 22.65 & 21.95 & 30.04 & 19.57 \\
\hline 9 & 55.81 & 10.31 & 68.01 & 11.86 \\
\hline 10 & 48.58 & 14.77 & 61.97 & 7.99 \\
\hline 11 & 24.56 & 22.67 & 43.98 & 16.49 \\
\hline 12 & 60.63 & 7.71 & 70.08 & 13.25 \\
\hline 13 & 23.65 & 28.51 & 49.04 & 15.11 \\
\hline
\end{tabular}

DE: Dissolution efficiency; MDT: Mean dissolution time, Data given as mean \pm S.D. $(n=3)$

\section{Mathematical modelling}

As per ANOVA results, all the polynomial equations were found statistically significant $\left({ }^{*} \mathrm{p}<0.05\right)$. The values obtained showed HPB has more effect on both responses (\%DE and MDT) compared to PVP K30.

\section{Response surface analysis}

Figures $4 \mathrm{~A}$ and $7 \mathrm{~A}$ represents the 3 -dimensional response surface plots, while $4 \mathrm{~B}$ and $7 \mathrm{~B}$ are the corresponding contour plots for each response \% R by Kneading method and Spray drying method. Figures $5 \mathrm{~A}$ and $8 \mathrm{~A}$ represents the 3-dimensional response surface plots, while $5 \mathrm{~B}$ and $8 \mathrm{~B}$ are the corresponding contour plots for each response \% DE by Kneading method and Spray drying method, exhibited that dissolution efficiency vary in an ascending order but in a non-linear manner with an increase in amount of each polymer. The contour plot showed that HPB has comparatively greater influence on dissolution efficiency than PVP K30.

Figures $6 \mathrm{~A}$ and $9 \mathrm{~A}$ represents the 3 -dimensional response surface plots, while $6 \mathrm{~B}$ and $9 \mathrm{~B}$ are the corresponding contour plots for each response MDT by Kneading method and Spray drying method. Effect of HPB and PVP K30 on MDT is depicted in figure 6 and 9. Inverse relation was observed on mean dissolution time by use of HPB and PVP K30. High concentration of HPB showed minimum MDT; whereas high level of PVP K30 increased MDT slightly.
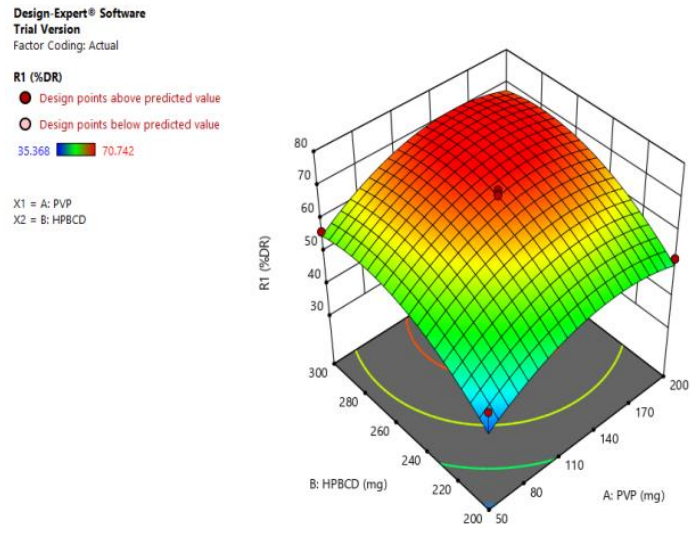
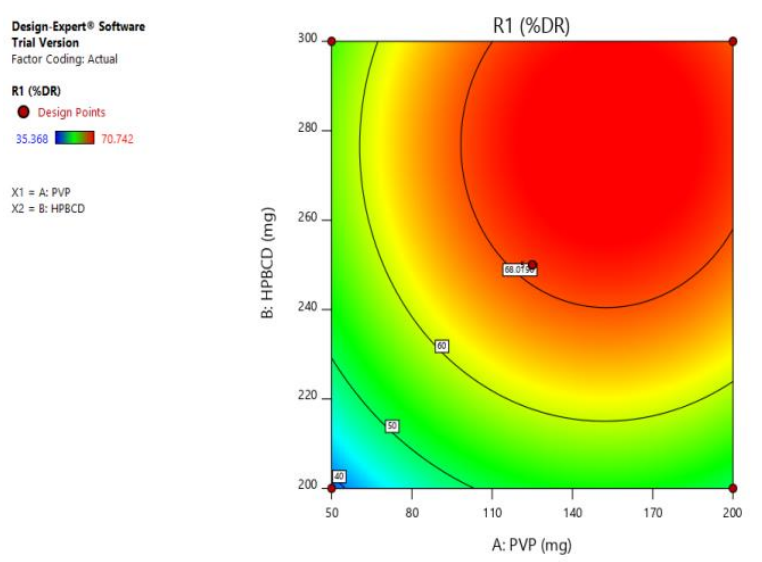

Figure 4: Effect of PVP K30 and HPB on \%DR of Kneading method, A) 3D Graph, B) contour graph, PVP: polyvinyl pyrrolidone; HPBCD: Hydroxypropyl $\beta$-cyclodextrin, \%DR: Percent drug release. 

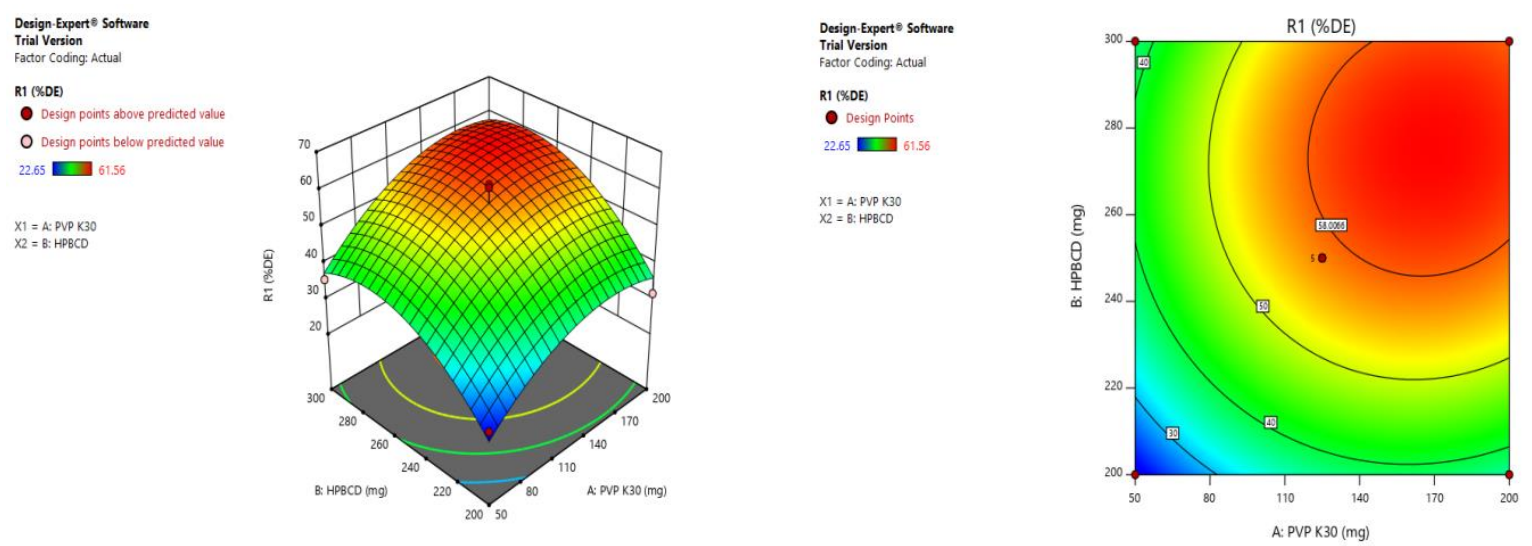

Figure 5: Effect of PVP K30 and HPB on \%DE of Kneading method, A) 3D Graph, B) contour graph, PVP: polyvinyl pyrrolidone; HPBCD: Hydroxypropyl $\beta$-cyclodextrin, \%DE: Percent dissolution efficiency.
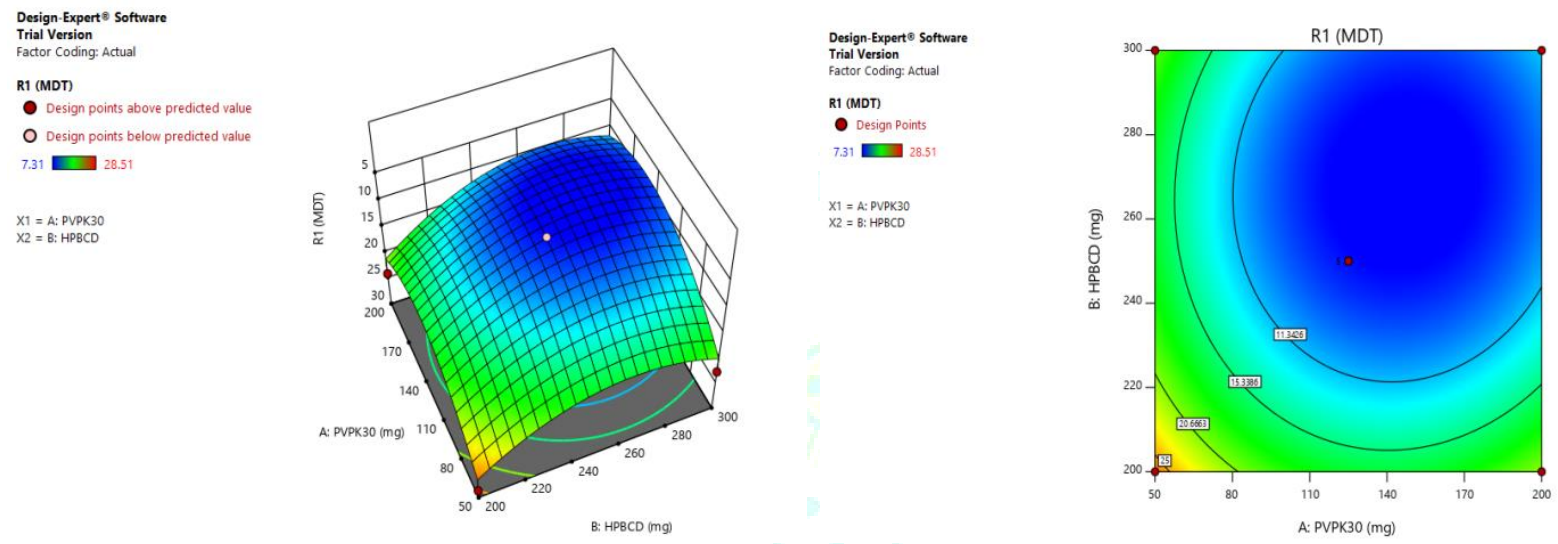

Figure 6: Effect of PVP K30 and HPB on MDT of Kneading method, A) 3D Graph, B) contour graph, PVP: polyvinyl pyrrolidone; HPBCD: Hydroxypropyl $\beta$-cyclodextrin, MDT: Mean dissolution time.
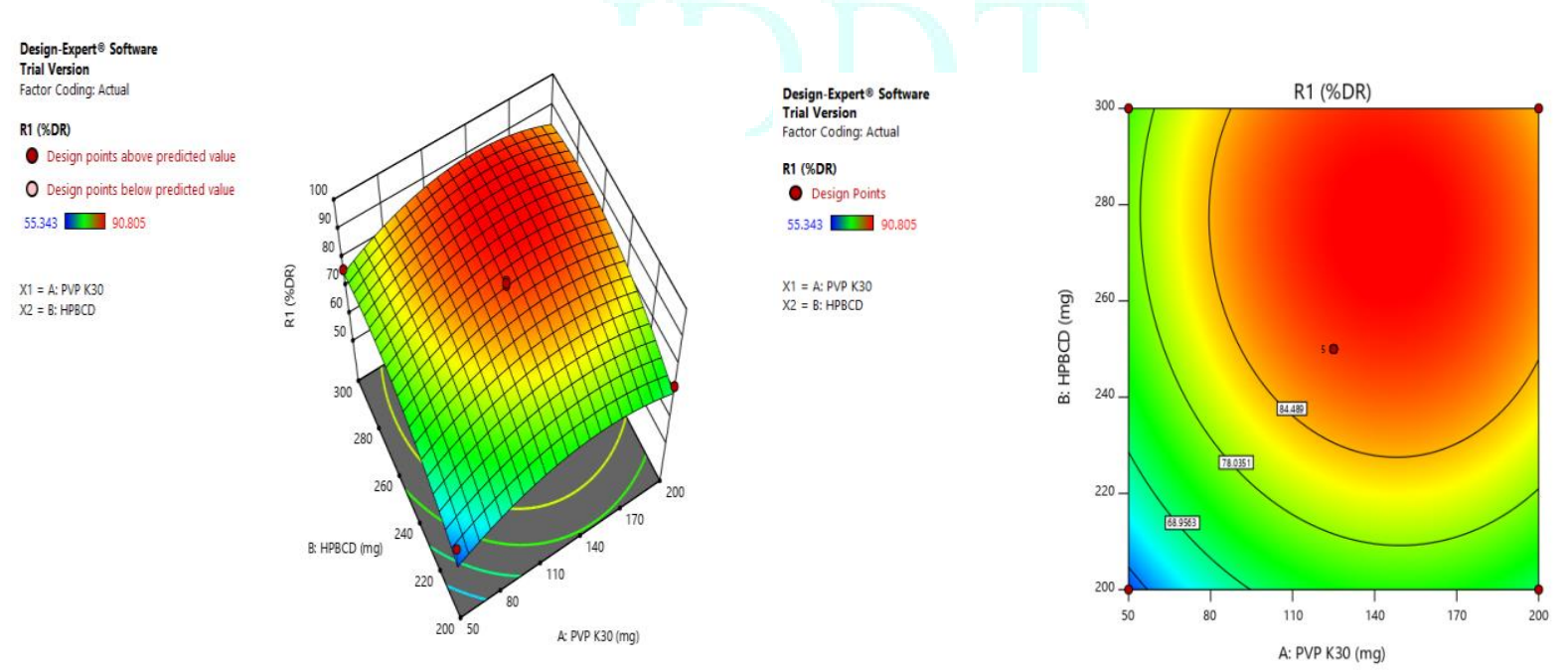

Figure 7: Effect of PVP K30 and HPB on \%DR of Spray drying method, A) 3D Graph, B) contour graph, PVP: polyvinyl pyrrolidone; HPBCD: Hydroxypropyl $\beta$-cyclodextrin, \%DR: Percent drug release. 

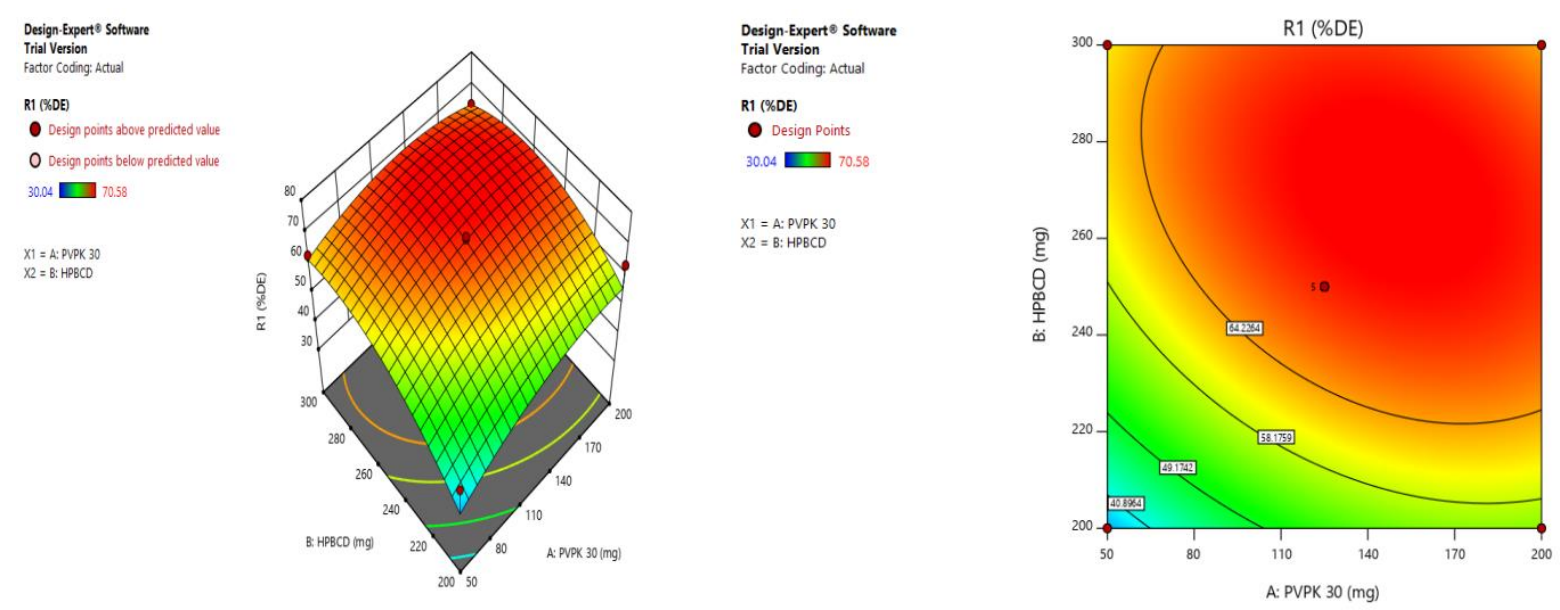

Figure 8: Effect of PVP K30 and HPB on \%DE of Spray drying method, A) 3D Graph, B) contour graph, PVP: polyvinyl pyrrolidone; HPBCD: Hydroxypropyl $\beta$-cyclodextrin, \%DE: Percent dissolution efficiency.
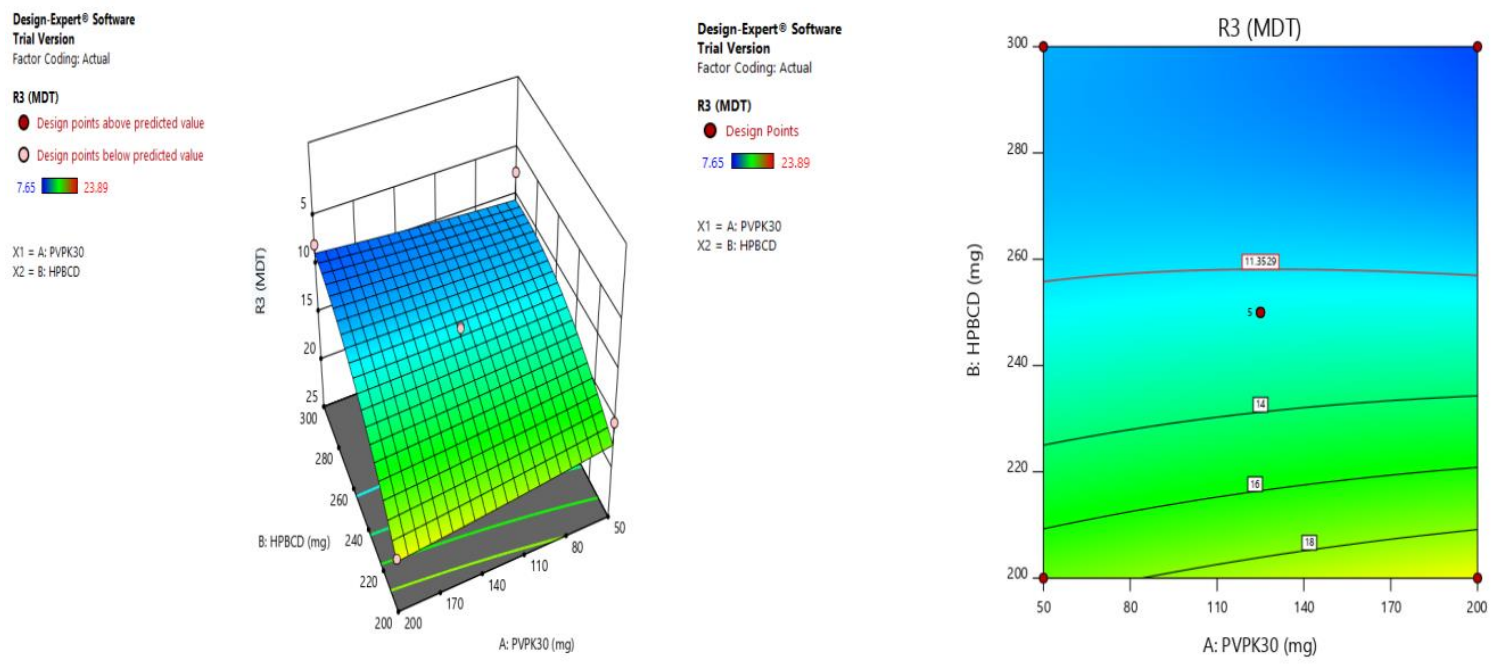

Figure 9: Effect of PVP K30 and HPB on MDT of Spray drying method, A) 3D Graph, B) contour graph, PVP: polyvinyl pyrrolidone; HPBCD: Hydroxypropyl $\beta$-cyclodextrin, MDT: Mean dissolution time.

\section{Flow property measurment}

The preparation of essentially all dosage forms involves the handling of solid materials. The flow properties of solids have a great impact on various steps in pharmaceutical operations like sieving, micronizing, pouring, mixing, grinding, pneumatic conveying, drying compaction, etc. Hence, this study was carried out on ETO and optimized ternary system of ETO, PVP K30 and HPB. The result of the study revealed that there was marked improvement in flow properties of CLT in the optimized ternary system as compared to plain CLT (Table 3).

Table 3: Study of flow properties

\begin{tabular}{|l|l|l|l|}
\hline Sr. No. & Parameters & CLT & Optimized ternary system \\
\hline 1 & Bulk density $\left(\mathrm{gm} / \mathrm{cm}^{3}\right)$ & 0.60 & 0.70 \\
\hline 2 & Tapped density $\left(\mathrm{gm} / \mathrm{cm}^{3}\right)$ & 0.94 & 0.80 \\
\hline 3 & Compressibility index $(\%)$ & 22.12 & 12.5 \\
\hline 4 & Hausner's ratio & 1.60 & 1.14 \\
\hline 5 & Angle of repose $(\stackrel{\mathrm{o}}{)}$ & 37.48 & 19.64 \\
\hline
\end{tabular}

\section{Characterization of optimized ternary system}

To confirm the formation of solid complex and its amorphous nature, the ternary system of CLT-PVP-HPB obtained by spray drying process was characterised by FTIR, DSC, XRD and particle size analysis. 


\section{a) FTIR spectroscopy}

CLM show characteristic peaks of O-H Stretching at $3476 \mathrm{~cm}$ $1, \mathrm{C}=0$ Stretching at $1728 \mathrm{~cm}-1, \mathrm{C}-\mathrm{CH} 3$ Stretching at 1458 $\mathrm{cm}-1, \mathrm{C}-0$ Bending at $1372 \mathrm{~cm}-1$. FT-IR study revealed that there was no major change in the position of peak values obtained in the Clarithromycin alone and in formulation of Clarithromycin solid dispersion with Excipients, which shows that there was no interaction between Clarithromycin and Excipients. Results are shown in figures 10.
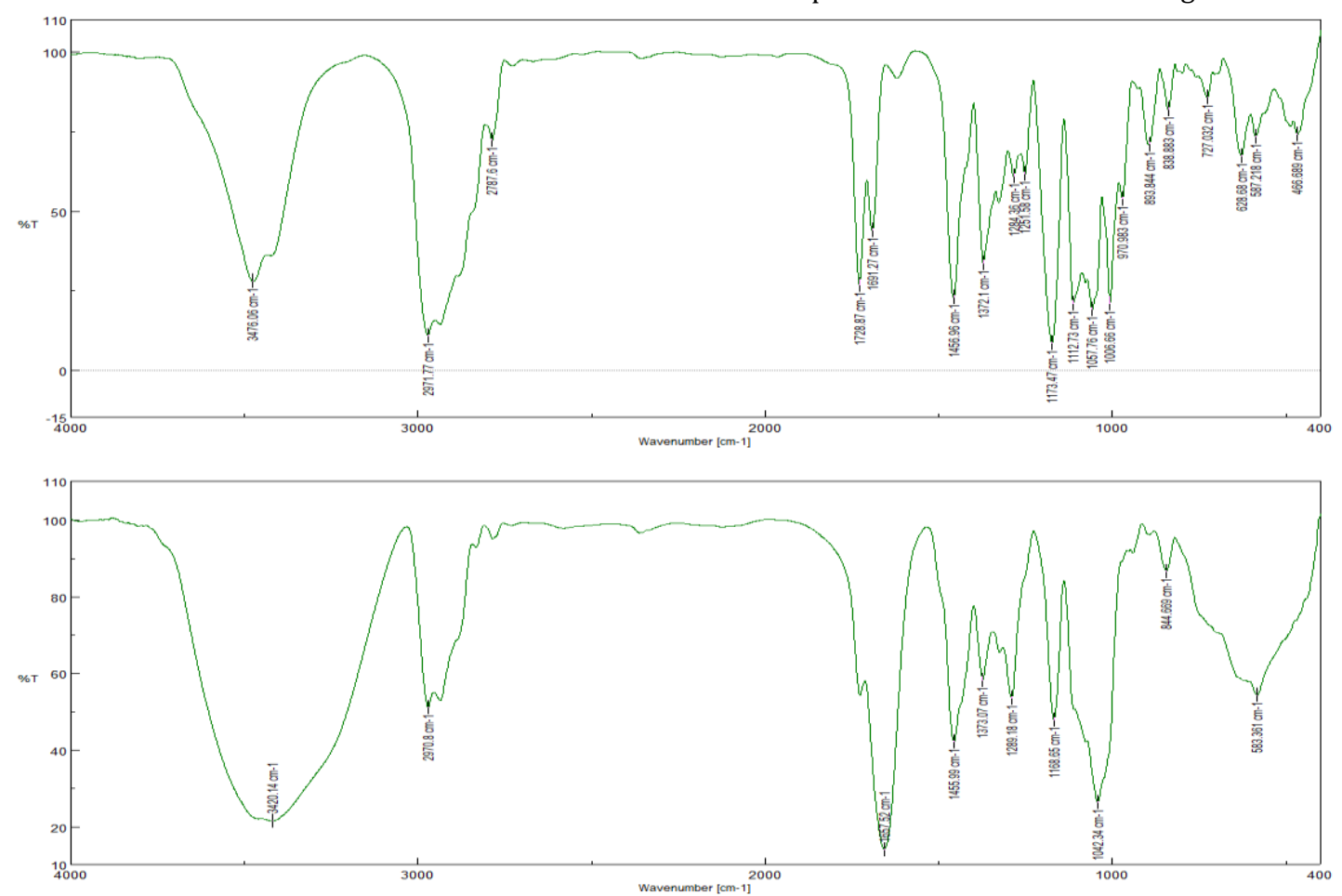

Figure 10: FTIR spectra of A: Pure CLT, B: Optimized ternary system

\section{b) Differential scanning calorimetry (DSC)}

The sharp endothermic peak at $224.5^{\circ} \mathrm{C}$ in plain CLT sample indicated the melting point of the drug as shown in fig.11. DSC thermogram of the CLT-PVP-HPBCD complex did not show any peak at CLT's melting point $\left(224.59^{\circ} \mathrm{C}\right)$. This represents that drug is enclosed in HPBCD cavity to form inclusion complex and it has converted into the amorphous state $^{17}$.
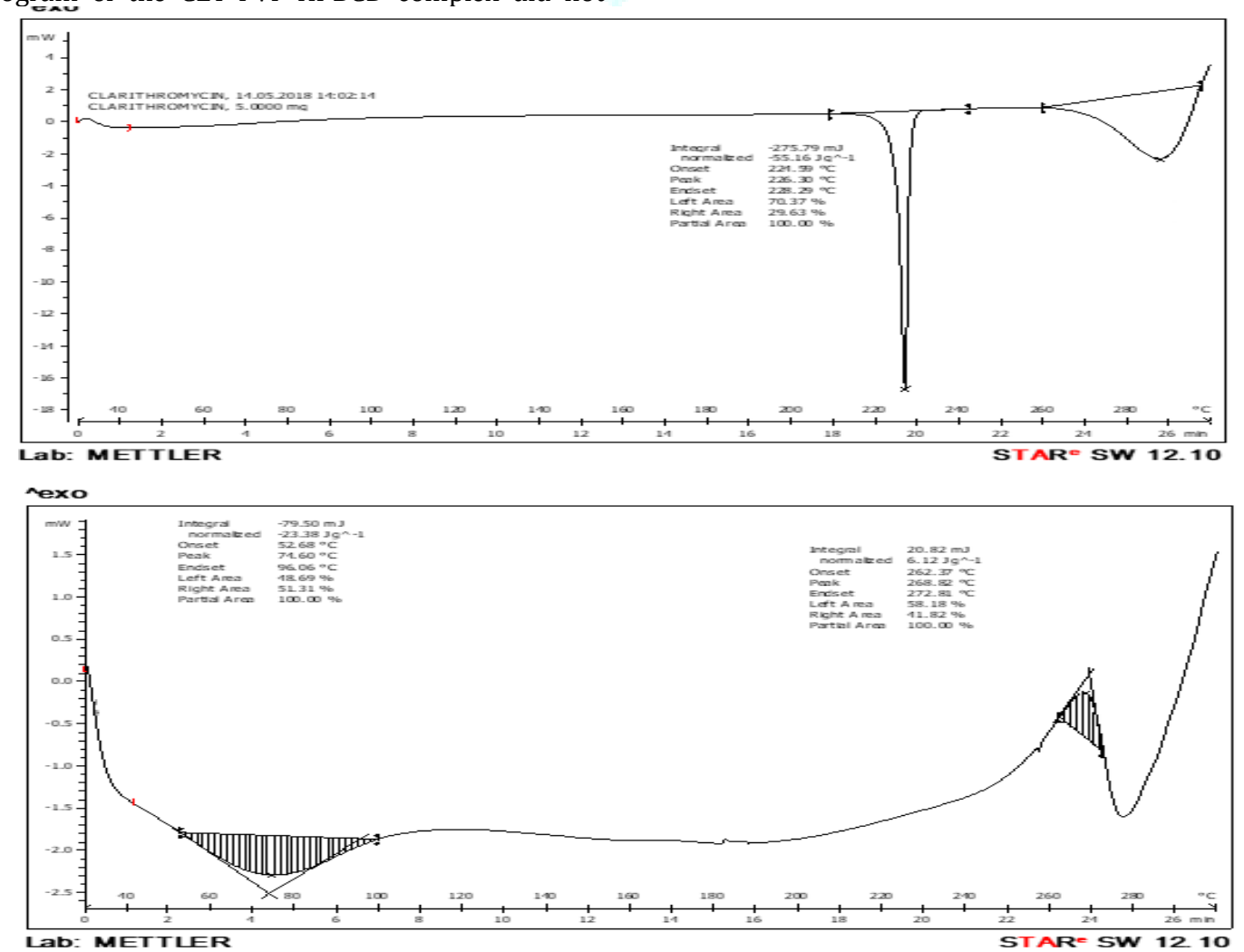

Figure 11: DSC thermogram of A: Pure CLT, B: Optimized ternary system 


\section{c) X-ray diffraction (XRD)}

The powder X-ray diffractogram of Clarithromycin exhibited intense peaks and confirmed high crysatallanity. CLT-PVP K30-HPB complex was found amorphous in nature (fig.12).
The Optimized Solid Dispersion showed peaks of Clarithromycin having less intensity than pure form indicating loss of crystallinity. The difference in number and shape of peaks in these two diffractograms confirmed complex formation.
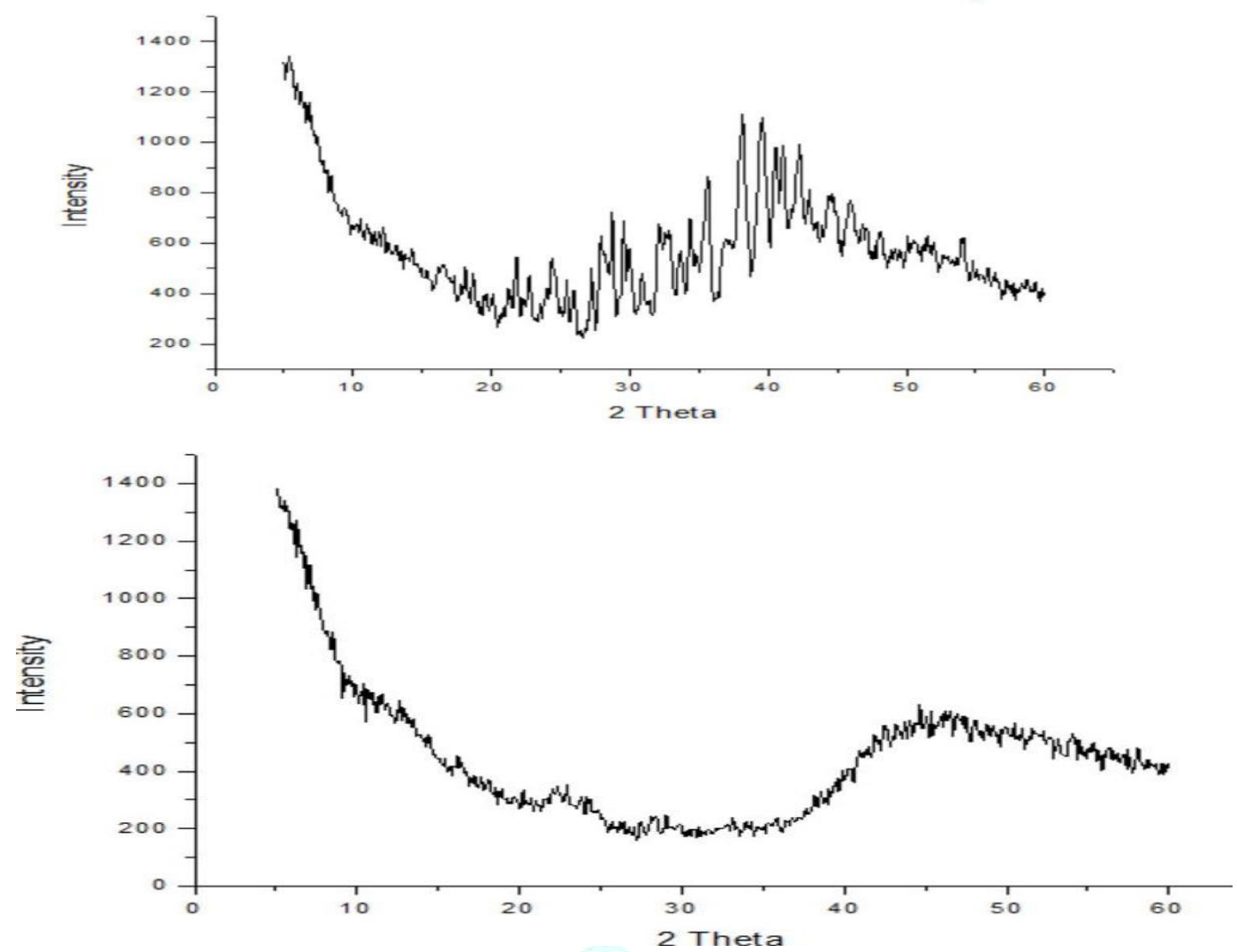

Figure 12: X-ray diffraction of A: Pure CLT, B: Optimized ternary system

\section{d) Particle size analysis:}

Analysis of particle size was performed on an optimised ternary system (CLT: PVP K30: HPB). Particle size of the selected formulation was found to be $312 \mathrm{~nm}$.

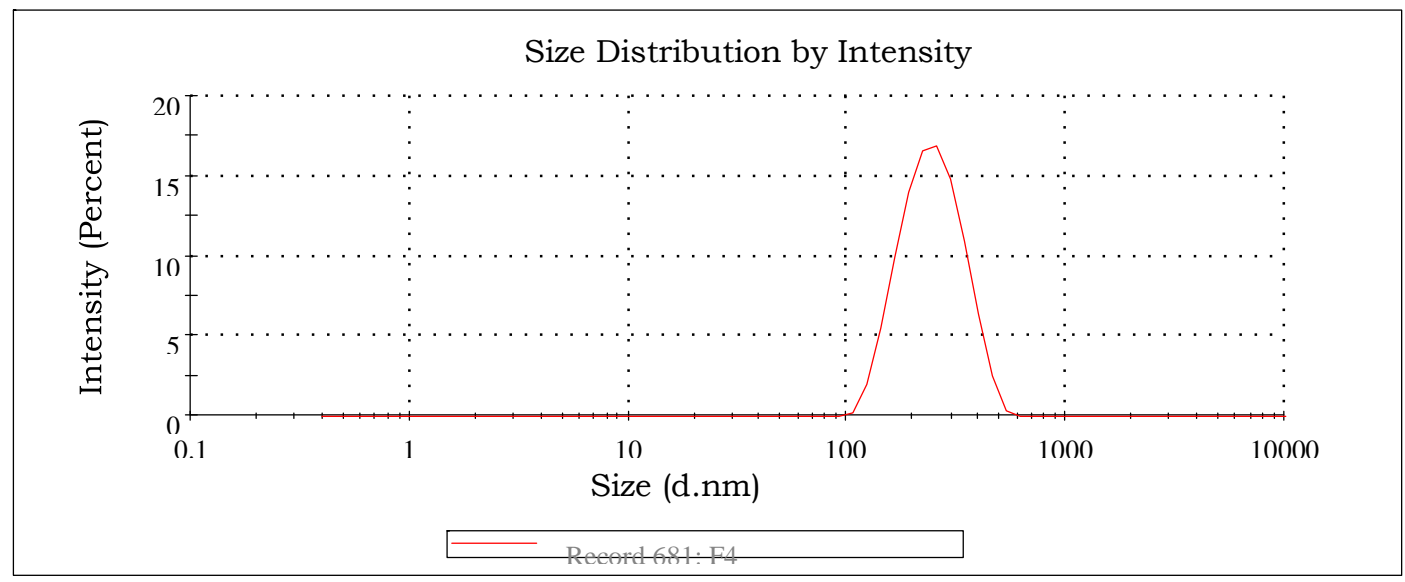

Figure 13: Particle size analysis of optimized ternary system 


\section{CONCLUSION}

Predictable dissolution attribute for CLT can be successfully achieved by DoE approach in the ternary system. Effects of PVP K30 and HPB on the DR, DE and MDT of CLT can be evaluated and optimized effectively using DoE approach. Results of the present study concluded that combined use of PVP K30 and HPB with the multivariate approach is an effective method for formulating a ternary system of CLT by spray drying method.

\section{ACKNOWLEDGEMENT}

The authors are thankful to Dr D. Y. Patil College of Pharmacy, Pune for providing the necessary facility and infrastructure to conduct this research work. The authors are grateful to Alembic pharmaceuticals Ltd., Vadodara and Lupin research park, Pune for providing the gift samples.

\section{CONFLICT OF INTEREST}

Authors explicitly declare that there is no conflict of interest.

\section{REFERENCES}

1. Manimaran V, Damodharan N, Mothilal M, Rajkumar K, Chalackal RM. Enhancement of dissolution rate of glibenclamide by solid dispersion technology. Int J Curr Pharm Res; 2010; 2:14-7.

2. Kurmi R, Mishra DK, Jain DK. Solid dispersion: a novel means of solubility enhancement. J Crit Rev; 2015; 3:1-8.

3. Brewster ME, Loftsson T. Cyclodextrins as pharmaceutical Delivery Rev; 2007; 59:645-66.

4. Anil Shinde, Harinath N More. Solubilization of Poorly Soluble Drugs: A Review; 2007; 5(6).

5. Parmar SS, Mishra R, Shirolkar SV. Spherical agglomeration a novel approach for solubility and dissolution enhancement of simvastatin. Asian J Pharm Clin Res; 2016; 9:65-72.

6. Duchene D, Wouessidjewe D, Ponchel G. Cyclodextrins and carrier systems. J Controlled Release; 1999; 62:263-8.
7. Mendhe AA, Kharwade RS, Mahajan UN. Dissolution enhancement of poorly water-soluble drug by cyclodextrins inclusion complexation. Int J Appl Pharm; 2016; 8:60-5.

8. Shuang S, Choi MM. Retention behaviour and fluorimetric detection of procaine hydrochloride using carboxymethyl bcyclodextrin as an additive in reversed-phase liquid chromatography. J Chromatogr; 2001; A919:321-9.

9. Bhatt P, Narvekar P. Challenges and Strategies for Drug Transport across the Blood Brain Barrier. ARC Journal of Neuroscience. 2018; 3(3):17-21.

10. Rajewski RA, Stella VJ. Pharmaceutical applications of cyclodextrins. 2. In vivo drug delivery. J Pharm Sci; 1996; 85:1142-69.

11. Carrier RL, Miller LA, Ahmed I. The utility of cyclodextrin for enhancing oral bioavailability. J Controlled Release; 2007; 127:78-99.

12. Singh B, Kumae R, Ahuja N. Optimizing drug delivery system using systematic "Design of Experiments."Part I: Fundamental Aspects Critical Reviews ${ }^{\mathrm{TM}}$ in Therapeutic. Drug Carrier Systems; 2004; 22:27-105.

13. S. N. Hiremath, RK Raghavendra, F. Sunil, LS Danki, M V Rampure, PV Swamy, UV Bhosale. Dissolution enhancement of gliclazide by preparation of inclusion complexes with $ß$ cyclodextrin. Asian Journal of pharmaceutics; 2008; 73-76.

14. Amir A Shaikh, Praveen D Chaudhari, Sagar S Holkar. A design of experiment approach for Optimization and Characterization of Etodolac Ternary System Using Spray Drying. Int J Pharm Pharm Sci; 2017; 9(2):233.240.

15. Singh B, Dahiya M, Saharan V, Ahuja N. Optimizing drug delivery systems using systematic "Design of Experiments."Part II: Retrospect and Prospects Critical Reviews $^{\mathrm{TM}}$ in Therapeutic. Drug Carrier Systems; 2005; 22:215-93.

16. Al-Hamidi H, Edward AA, Mohammad MA, Nokhodchi A. To enhance the dissolution rate of poorly water-soluble drugs: glucosamine hydrochloride as a potential carrier in solid dispersion formulations. Colloids surf B; 2010; 76:170-8.

17. Costa FO, Souse JJ, Pais AA, Fomosinho SJ. Comparision of dissolution profile of ibuprofen pellets. J Controlled Release; 2003; 89:199-212. 